\title{
Avaliação de quarta geração em Centro de Atenção Psicossocial Álcool e Drogas
}

Fourth-generation evaluation in an Alcohol and Drugs Psychosocial Care Center Evaluación de cuarta generación en Centro de Atención Psicosocial Alcohol y Drogas

Paula Antunes Bezerra Nacamura ${ }^{1}$ (b) Maria Aparecida Salci ${ }^{1}$ (D)

Valéria Cristina Christello Coimbra ${ }^{2}$ (1) André Estevam Jaques ${ }^{1}$ (D) Mauro Belino Piratelli Filho ${ }^{1}$ (c) Jéssica dos Santos Pini ${ }^{1}$ (D) Camila Harmuch $^{1}$ (D) Marcelle Paiano ${ }^{1}$ (I)

1. Universidade Estadual de Maringá, Programa de Pós-Graduação em Enfermagem. Maringá, PR, Brasil.

2. Universidade Federal de Pelotas. Pelotas, RS, Brasil.
Autor correspondente:

Paula Antunes Bezerra Nacamura.

E-mail: palinhaa.a.b@hotmail.com

Recebido em 11/08/2021.

Aprovado em 17/11/2021.

\section{Resumo}

Objetivo: Avaliar qualitativamente as ações ofertadas por um Centro de Atenção Psicossocial Álcool e Drogas na perspectiva dos familiares e dos usuários do serviço. Método: Estudo qualitativo, do tipo estudo de caso, desenvolvido pelo método de avaliação de Quarta Geração, nos meses de setembro de 2019 a março de 2020. Os dados foram coletados por meio da observação e de entrevista aberta individual e analisados pelo Método Comparativo Constante. Os informantes foram nove familiares e oito usuários do serviço. Para auxiliar na organização e na apresentação dos dados, utilizou-se o software ATLAS.ti 9.0.18. Resultados: Ambos os grupos de interesse ressaltaram positivamente a gratuidade do tratamento, o acolhimento, o apoio, as informações ofertadas pela equipe por meio dos grupos, a reinserção social e a oferta de medicação. Além disso, psicólogos e assistentes sociais foram citados como profissionais de maior proximidade. $\mathrm{O}$ acesso foi citado com ambiguidade pelos grupos de interesse, e as fragilidades incluíram a continuidade do cuidado pelos pontos da rede psicossocial. Considerações finais e implicações para a prática: O estudo reconhece a eficácia do serviço, no entanto, necessita aprimoramento na articulação das ações interprofissionais e intersetoriais entre os demais pontos de atenção e a inclusão da família no tratamento.

Palavras-chave: Avaliação em Saúde; Centros de Tratamento de Abuso de Substâncias; Família; Serviços de Saúde Mental; Usuários de Drogas.

\section{Abstract}

Objective: To qualitatively evaluate the actions provided by an Alcohol and Drugs Psychosocial Care Center from the perspective of family members and service users. Method: This is a qualitative case study developed using the fourth-generation evaluation method from September 2019 to March 2020. Data were collected through observation and individual open interviews and analyzed by the constant comparative method; nine family members and eight service users were informants. The ATLAS ti 9.0.18 software was used to help organize and present the data. Results: Both focus groups highlighted positively the free treatment, reception, support, and information provided by the team through the groups, social reintegration, and providing medication. In addition, psychologists and social workers were cited as the professionals that the participants felt the closest to. Access to care was cited with ambiguity by the focus groups, and the weaknesses included the continuity of care by the points of the psychosocial network. Final considerations and implications for practice: This study recognizes the effectiveness of the service, although it requires improving the articulation of interprofessional and intersectoral actions among the other points of care and including the family in the treatment.

Keywords: Health Evaluation; Substance Abuse Treatment Centers; Family; Mental Health Services; Drug Users.

\section{Resumen}

Objetivo: Evaluar cualitativamente las acciones ofrecidas por un Centro de Atención Psicosocial Alcohol y Drogas en la perspectiva de los familiares y usuarios del servicio. Método: Estudio cualitativo, del tipo de estudio de caso, desarrollado por el método de evaluación de Cuarta Generación, entre los meses de septiembre de 2019 hasta marzo de 2020. Los datos se recogieron mediante la observación y la entrevista abierta individual y se analizaron mediante el Método Comparativo Constante. Los informantes fueron nueve familiares y ocho usuarios del servicio. Se utilizó el software ATLAS.ti 9.0.18 para ayudar a organizar y presentar los datos. Resultados: Ambos grupos de interés destacaron positivamente la gratuidad del tratamiento, la acogida, e apoyo y la información ofrecida por el equipo a través de los grupos, la reinserción social y la oferta de medicación. Además, los psicólogos y los trabajadores sociales fueron mencionados como profesionales de mayor proximidad. El acceso fue aludido con ambigüedad por los grupos de interés, y entre los puntos débiles estaba la continuidad de la atención por parte de los puntos de la red psicosocial. Consideraciones finales e implicaciones para la práctica: El estudio reconoce la eficacia del servicio sin embargo, necesita mejorar la articulación de las acciones interprofesionales e intersectoriales entre los demás puntos de atención y la inclusión de la familia en el tratamiento.

Palabras clave: Evaluación de la salud; Centros de Tratamiento de Abuso de Sustancias; Familia; Servicios de Salud Mental; Usuarios de Drogas. 


\section{INTRODUÇÃO}

Em 2017, por meio da Portaria GM/MS 3.588, houve a reorganização de alguns pontos da Rede de Atenção Psicossocial (RAPS), com o retorno dos hospitais psiquiátricos como integrantes da rede, dando início a um processo de desmonte do que foi construído ao longo de décadas no âmbito da Reforma Psiquiátrica $(\mathrm{RP})^{1}$. Essa mudança, juntamente às demais ocorridas na política de saúde mental, representa grande risco aos serviços de base comunitária, principalmente aos centros de tratamento de abuso de substâncias.

Em 2019, as modificações nas diretrizes da Política Nacional sobre Drogas legitimaram as comunidades terapêuticas como estratégia de tratamento para usuários de substância, priorizando a abstinência em detrimento da redução de danos e desconsiderando os serviços de atenção psicossocial como principais locais de atendimento e de tratamento dessa população².

Tais mudanças implicam a reversão de direitos conquistados no contexto da RP, enfraquecendo as atividades desenvolvidas até então pelos Centros de Atenção Psicossocial (CAPS), os quais apontam a relevância da participação social aos cuidados em saúde mental, a priorizar a psiquiatria em detrimento de outros campos de conhecimento, da clínica ampliada e do trabalho em equipe interdisciplinar ${ }^{3}$.

Apesar do cenário desfavorável, o CAPS ad, como um serviço especializado e territorial inserido na RAPS, prioriza o cuidado e a assistência direcionada aos usuários de drogas e a seus familiares. Nesse contexto, a família deve ser vista como parceira singular e participativa no processo, mas que também necessita de cuidados ${ }^{4}$.

Dessa maneira, a participação dos familiares e dos usuários, na construção do cuidado, favorece não apenas o resultado e a aproximação entre os grupos envolvidos, mas também possibilita a reinserção social e a autonomia do cuidado, valorizando o atendimento ao usuário e o contexto familiar e contribuindo para a construção do conhecimento a partir das experiências vividas ${ }^{5,6}$.

O compartilhamento de experiências entre profissionais, usuários e familiares pode inspirar a construção de novas possibilidades na área da atenção psicossocial, por meio da construção coletiva, na convicção de uma prática assistencial que considere o indivíduo na sua totalidade ${ }^{7}$.

Para isso, as avaliações em serviço tornam-se importantes instrumentos na busca de respostas, constituindo-se em estratégia central para a verificação de aspectos da qualidade do cuidado à saúde, que é fundamental para a ampliação e a qualificação do acesso e do atendimento à população ${ }^{8,9}$.

Nesse sentido, desponta a necessidade de investir-se em uma proposta participativa, orientada por um modelo de avaliação em saúde que dá voz aos grupos envolvidos e que busca metodologias sistêmicas, dinâmicas e interativas, proporcionando o protagonismo necessário a todos os atores institucionais e o aprimoramento do serviço ${ }^{8,9}$.

Assim, considerando a importância da participação comunitária/ social para a construção do cuidado na atenção psicossocial, em especial na atenção ao consumo de álcool e de outras drogas, este estudo tem por objetivo avaliar qualitativamente as ações ofertadas por um CAPS ad na perspectiva dos familiares e dos usuários do serviço.

\section{MÉTODO}

Trata-se de um estudo de abordagem avaliativa e qualitativa, pertencente a um projeto de dissertação intitulado "Avaliação de Quarta Geração em um Centro de Atenção Psicossocial Álcool e Drogas", apoiado no referencial teórico-metodológico da Avaliação de Quarta Geração ${ }^{10}$. Essa avaliação possui uma abordagem hermenêutico-dialética, de cunho construtivista e responsivo.

O estudo foi realizado no CAPS ad de um município de médio porte do Sul do país. Nesse serviço, as atividades realizadas englobam oficinas terapêuticas, grupo psicopedagógico, grupo de educação em saúde e orientações às famílias. Ele é constituído por uma equipe multiprofissional que realiza acolhimento ao usuário, ou ao familiar, o qual procura o serviço por demanda espontânea ou é encaminhado por outros dispositivos. Além disso, o CAPS ad possui horário de funcionamento de segundafeira a sexta-feira, das 7 às 21 horas.

O estudo contou com a participação de dois grupos de interesse. O primeiro grupo foi formado por nove familiares que estavam em acompanhamento no CAPS ad, e o segundo grupo, por oito usuários em tratamento no CAPS ad. Foram incluídos no estudo familiares e usuários maiores de 18 anos e que, nos três meses anteriores à pesquisa, tivessem sido atendidos pelo menos uma vez no serviço.

As informações foram coletadas entre os meses de setembro de 2019 e março de 2020, por meio da observação e de entrevista individual estabelecida pelo círculo hermenêutico-dialético (CHD). As observações de campo totalizaram 218 horas, sendo registradas em um diário de campo.

O convite aos participantes ocorreu de forma individual, e a entrevista foi realizada ao término do atendimento agendado no serviço, em sala reservada e privativa. As entrevistas foram orientadas pelo $\mathrm{CHD}$, sendo que o pesquisador selecionou o respondente inicial (R1) de cada grupo, definido a partir daquele de maior assiduidade nos atendimentos no período de observação do serviço.

Inicialmente, aplicaram-se duas questões norteadoras: "Como funciona o CAPS ad em que você é atendido?" e "Quais as potencialidades e as fragilidades do CAPS ad?". Após o depoimento de $\mathrm{R} 1$, as reivindicações, as preocupações e as questões (RPQs) propostas foram analisadas, dando origem à construção inicial (C1). Para o próximo respondente (R2), que foi indicado pelo R1, foram introduzidas as mesmas questões norteadoras e a construção inicial (C1), para que comentasse sobre elas. Esse processo foi repetido até que o círculo se fechasse sem novas indicações ou com construções que não apresentassem dados novos ${ }^{10}$.

O CHD foi concluído quando as RPQs não originaram dados novos para a pesquisa. Assim, seguindo esse critério, deliberouse pela conclusão dos CHDs dos familiares com a entrevista 
do nono respondente (R9), e dos usuários com a entrevista do oitavo respondente (R8). As entrevistas foram gravadas em áudio e transcritas na íntegra e foram analisadas com a utilização do Método Comparativo Constante, que permite que os dados sejam analisados concomitantemente à sua coleta ${ }^{10}$.

Após a finalização dessa etapa, não foi possível a realização da sessão de negociação, momento em que o conjunto de questões pode ser consensualmente validado pelos entrevistados ou modificado agregando novas informações ${ }^{10}$, em virtude da pandemia de Covid-19 e das restrições impostas por ela. Dessa forma, os dados apresentados neste estudo são provenientes das entrevistas realizadas por meio do CHD dos dois grupos de interesse.

Para auxiliar na organização e na apresentação dos dados, utilizou-se o software ATLAS.ti 9.0.18, a partir das ocorrências/ recorrências de termos e de expressões que estão distribuídos nos vários depoimentos analisados. O software oferece a possibilidade da geração de uma nuvem de palavras dos documentos ligados à unidade hermenêutica e permite a exibição visual da frequência das palavras, o que facilita a organização do conteúdo.

O estudo seguiu os preceitos éticos estabelecidos pelas Resoluções 466/12 e 510/2016 do Conselho Nacional de Saúde, com aprovação pelo Comitê Permanente de Ética em Pesquisa com Seres Humanos (CAAE 08929519.7.0000.0104). Os participantes foram esclarecidos sobre os objetivos do estudo, sobre os procedimentos de coleta de dados, os riscos e os benefícios e assinaram o Termo de Consentimento Livre e
Esclarecido. Para manter o anonimato dos participantes, eles foram identificados utilizando-se a letra "U" para usuários e a letra " $F$ " para familiares, seguidas de um número sequencial correspondente à ordem de realização das entrevistas.

\section{RESULTADOS}

As avaliações realizadas pelos grupos de interesse constituídos pelos familiares e pelos usuários resultaram em dados organizados e apresentados na Figura 1, a partir da ocorrência e da recorrência de informações distribuídas nos depoimentos.

Em consonância com o método comparativo constante, fol possível a construção de duas categorias. A primeira é "Ações ofertadas pelo CAPS ad e sua função na Rede de Atenção Psicossocial", sendo destacadas as seguintes palavras: "remédio", "tratamento", "Caps", "medicação", "assistente social", "psicólogo", "horário" e "problema". Já a segunda, "Ações do CAPS que impactam no tratamento: perspectiva do usuário e do familiar", destacando-se as palavras "acompanhamento", "reunião", "aprender", "necessidade", "orientar", "família”, "ajudar", "começar", "falar" e "conseguir".

1. Ações ofertadas pelo CAPS ad e sua função na Rede de Atenção Psicossocial

Ambos os grupos de interesse avaliaram o CAPS ad como um serviço de fundamental importância para o tratamento de pessoas em uso de álcool e de outras drogas. Alguns aspectos do serviço foram ressaltados de forma positiva, como a garantia

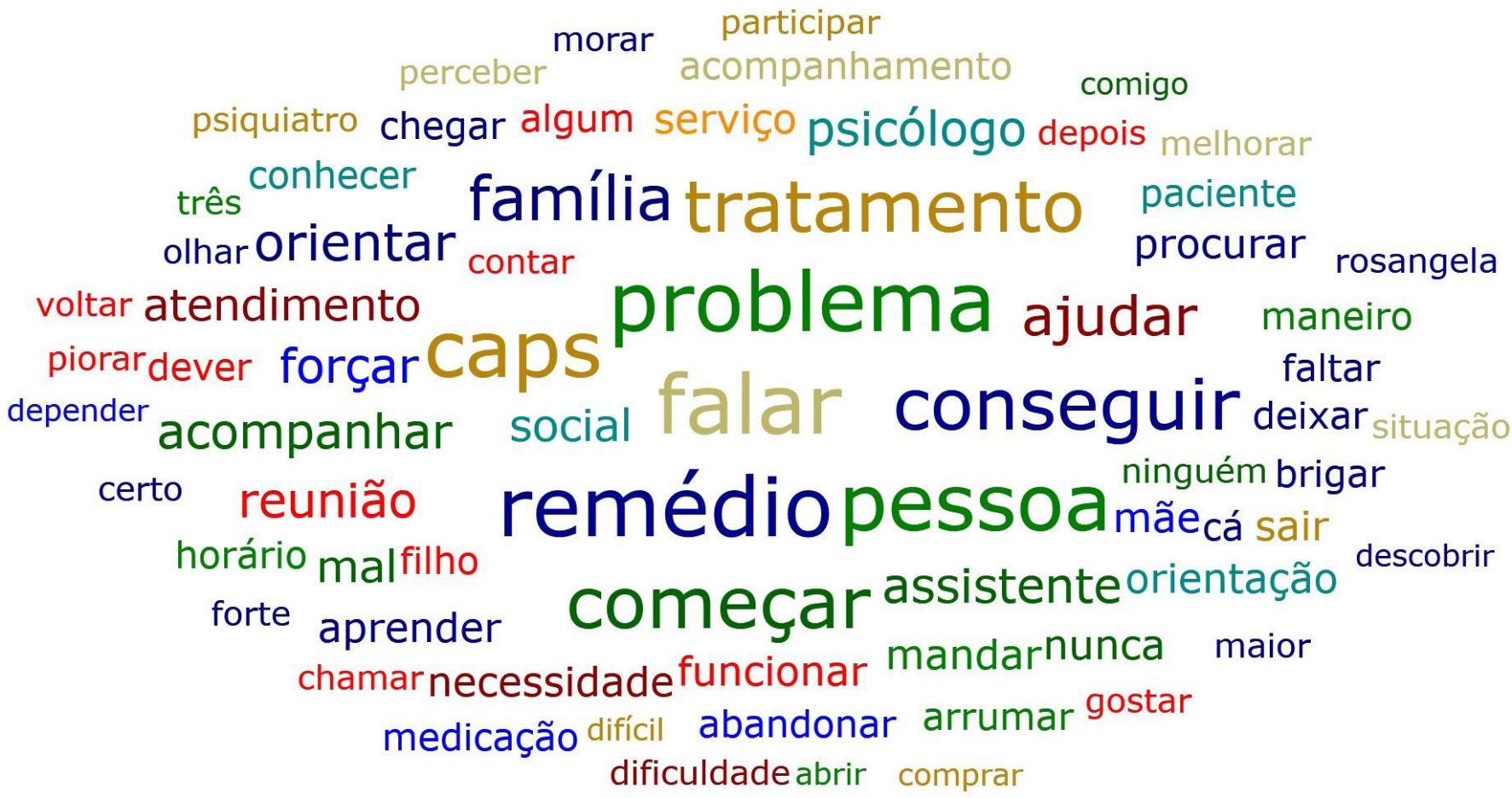

Figura 1. Nuvem de palavras: Avaliação do Centro de Atenção Psicossocial Álcool e Drogas por usuários e familiares. Elaborada pelos autores a partir dos dados da pesquisa no software Atlas.ti. Brasil, 2021. 
da integralidade e a gratuidade do tratamento e da medicação fornecida aos pacientes que estejam inseridos no plano de cuidados.

Se fosse para a gente pagar, continuar pagando o tratamento dele, a gente não tinha condições (F1).

Até agora não vi outra coisa melhor, que desse um resultado assim. Sem aquele custo [...], sem cobrar por isso. É excelente, humano (U1).

Tem a farmácia, onde eu pego o remédio, se a gente fica doente ou entra em crise, tem internamento ali no Caps III. Então, é uma estrutura muito boa, porque antes ele levava a gente para o sanatório (U5).

A equipe multiprofissional é identificada pelos usuários como positiva pelo suporte oferecido durante a prestação do cuidado. Destaca-se que os familiares mencionaram a assistente social e o psicólogo como os profissionais de maior contato.

Eles não tratam a gente como qualquer pessoa, eles tratam a gente como paciente, eles entendem o problema da gente, sabem que a gente tem problema, tem dificuldade, e eles sanam essas dificuldades (U5).

Passei portodos, e é assim uma vasta equipe de profissionais, e sempre fui muito bem amparado e atendido (U4).

Olha, nós, no caso que somos família, nós passamos por esse grupo da saúde (com assistente social) e pelo atendimento psicológico também. Então, eu vejo que é mais uma ajuda, no nosso caso é, é mais uma ajuda psicológica (F5).

O acesso dos usuários e dos familiares ao serviço trouxe uma avaliação ambígua, sendo compreendida como positiva pela disponibilidade do horário estendido, o que possibilita o comparecimento dos envolvidos sem prejuízos de suas atividades profissionais. No entanto, a localização geográfica do serviço foi avaliada negativamente, considerando que o CAPS ad está localizado em um bairro com elevada movimentação de consumo e de venda de drogas.

O horário aqui é bom, esse horário que eu venho, eu saio do serviço e venho cá, para mim é um horário conveniente, que está fora do horário do expediente de trabalho (F6).

A nossa maior dificuldade na realidade é a distância, que nós moramos do outro lado da cidade. A gente sai do trabalho já vem direto para cá. $E$ a gente tem acompanhado ele também, a gente vem junto, é um tanto quanto longe (F5).

Parece que o movimento de pacientes caiu com relação da onde era (endereço antigo), eu não sei se é a questão geográfica do local, porque aqui nós estamos num local em que, aparentemente, no passado ou até no presente, tem muitos pontos de drogas (U3).

Os familiares apontaram fragilidades na continuidade do cuidado pelos demais pontos da RAPS e o próprio desconhecimento dos serviços que a compõem. Soma-se a isso a não priorização desses serviços em proporcionar o atendimento solicitado pelo CAPS, principalmente quando se trata de cuidado psicológico à família.

Nós sabemos que existe, mas... não foi passado como funciona a rede (de saúde mental) (F5).

Às vezes tem situação que o filho está com violência, está violento, o que que faz numa situação dessa?! Chama o Samu, chama a polícia, chama não sei o quê?! (F6).

Ela indicou uma psicóloga, que tem que ser o tratamento através da unidade básica, ela me indicou num dia e no dia seguinte eu já fui, aí foi agendado lá que iam entrar em contato comigo, só que isso foi dia 21 de janeiro, eu fui lá dia 22, hoje, já estamos em março, de 21 de janeiro até 3 de março ela não me chamou ainda (F8).

A assistente social me encaminhou ali para o postinho de saúde, mas lá demora muito, que até agora não saiu (o atendimento) nem para mim nem para minha neta (F2).

Em contrapartida, para os usuários, observa-se a centralização do cuidado nos serviços do CAPS ad, quando eles deveriam contar com a participação da atenção primária nos cuidados de base territorial.

Quando tem algum problema, igual esse agora que surgiu (prurido nas mãos), eu vou passar pelo clínico geral (do CAPS). Da outra, ela me receitou uma pomadinha muito boa. Então, hoje quando eu passar pelo psicólogo, eu vou falar para ele me encaminhar para o clínico geral, que já conhece o meu problema, já sabe, já acompanha (U-5).

2. Ações do CAPS que impactam no tratamento: perspectiva do usuário e do familiar

Os familiares e os usuários foram unânimes em reconhecer que o acolhimento realizado, tanto no primeiro dia de atendimento quanto nos demais, incluindo as visitas domiciliares, é essencial para o seguimento do tratamento. Nessa perspectiva, a humanização oriunda do acolhimento pelos profissionais é avaliada positivamente pelos dois grupos de interesse.

Olha, este acompanhamento do CAPS, para mim, ele tem sido muito bom, como eu já falei, fui bem acolhida aqui, estou sendo. A assistente social é muito boa, ela sempre foi na minha casa, ela sempre mandou passe para eles virem aqui (F7). 
Eu me sinto muito bem acolhido. Nesse sentido, eles são muito atenciosos e muito amorosos, são profissionais dedicados, cada um na sua área (U4).

Todo momento que eu voltei eu fui super bem recebido, chamado pelo nome, os meus dados estavam todos no sistema, todos anotados, meus prontuários, eles já me conheciam, já sabiam da minha situação, e me acolheram super bem. É nessa parte, fantástico (U3).

As orientações realizadas pelos profissionais, nos grupos de apoio ou nos atendimentos individuais, foram destacadas pelos entrevistados, em virtude da qualidade das informações recebidas e dos direcionamentos realizados pelos profissionais que os conduzem.

Eu acho que as principais orientações é saber ter paciência e inteligência para lidar com o filho (F6).

Eles me dão todas as orientações, eles me pontuam, eu consigo perceber que não adianta só parar de usar drogas, mas você tem que, de fato, efetivar um tratamento (U3).

Foram objetivos, que a familia tinha que participar, e eles estão vindo, e às vezes eu olho assime falo: "poxa, poderia ter vindo antes", porque seria de grande valia, a maior dificuldade era o relacionamento familiar. Hoje, assim, eu tenho um relacionamento com meus pais que não tem como descrever, mudou da água para o vinho (U4)

Além disso, o apoio emocional ofertado aos familiares e aos usuários foi avaliado positivamente, considerando o enfrentamento das dificuldades no convívio de uma pessoa em uso de álcool e de outras drogas. As ações que estimulam a ressocialização e o retorno ao mercado de trabalho também foram citadas como positivas.

Me dá uma força, uma estrutura mais forte, porque a gente sozinha não consegue. Então, eu venho aqui na reunião de família, já faz mais de 2 anos, e é aqui que eu me fortaleço, que eu conto os meus problemas e ela me dá as orientações (F2).

Eles dão vale transporte, eles dão alimentação, eles dão acompanhamento, se a pessoa tem alguma dificuldade em casa eles encaminham para o serviço da assistente social para pegar cesta básica, para pegar vale alimentação, tudo isso (U5).

Para mim foi bom, porque eu estava com dificuldade para trabalhar, aí hoje eu já não tenho mais dificuldade. Ajudou no trabalho, mas na bebida não consegui parar (U8).

Para os familiares entrevistados, o serviço oferece esclarecimentos, recursos e ações necessárias para que o usuário dê continuidade ao tratamento, envolva-se e seja responsável pelo seu autocuidado.
Eu acho que se a pessoa tiver força de vontade, correr atrás, fazer tudo certinho, tomar os remédios certinho. Porque tem psiquiatra, tem psicólogo, tem as medicaçoes, tem a assistente social. Então, eu acho que supre sim, basta querer (F2).

Conforme essas coisas vão sendo colocadas de lado, o normal, o humano, o orgânico volta a funcionar, aí você passa a ter que ter vontade mesmo de continuar, porque ali começa uma briga, com dependência, você fica lutando com você mesmo. E nesse ponto, o CAPS é essencial porque tem a ajuda (U1).

Tem uma estrutura física e tem um grupo de profissional que trabalha aqui, se dedica, e hoje, dentro dessa cidade, só não tem um auxílio realmente se a pessoa não quiser, porque aqui você tem a possibilidade de psicóloga, psiquiatra, terapia ocupacional e assistente social, você tem o passe que você recebe para vir, para ir embora, então, se você quiser, funciona (U3).

Entre as ações ofertadas no tratamento ao uso de álcool e de outras drogas, as relações interpessoais, o vínculo e o apoio emocional entre profissionais e usuários/familiares são avaliados positivamente de forma a impactarem no tratamento, assim como no protagonismo do usuário para a eficácia de sua reabilitação.

\section{DISCUSSÃO}

Entre as ações ofertadas pelos CAPS ad, compreendem-se atividades voltadas para as pessoas que usam álcool e outras drogas e para seus familiares, estabelecidas sob a lógica interdisciplinar, da ressocialização e da participação social ao incluir familiares nos cuidados em saúde mental.

Entre os familiares e os usuários, foi consenso que uma das potencialidades do CAPS ad refere-se ao fornecimento de medicação como ferramenta indispensável para o tratamento do usuário. Nota-se que a medicação é vista como o principal caminho para o alcance do bem-estar e da capacidade de conviver em sociedade. Nesse sentido, é preciso cautela, pois, quando o assunto é medicação, há uma tendência à desresponsabilização de toda a equipe, designando o médico como único responsável pelas orientações ao tema ${ }^{11}$. Além disso, a primazia do modelo biomédico para o cuidado em saúde mental merece ser repensada, visto que parece contribuir para que os participantes sintam-se impedidos de uma convivência social satisfatória e reafirmem a centralidade da terapia medicamentosa para o cuidado de seus transtornos relacionados ao uso de substâncias ${ }^{12}$.

Quando se fala em equipe multiprofissional, sabe-se que, para atingir uma combinação interdisciplinar, é necessário que ela esteja pautada nas tecnologias leves, em uma intervenção terapêutica resolutiva e que estimule a autonomia do usuário. Assim, conforme é estabelecido o vínculo do profissional com o usuário, ele se torna corresponsável pelo acompanhamento desse usuário, seja no CAPS seja na atenção primária ${ }^{13}$. 
Na avaliação feita pelos usuários e pelos familiares, duas categorias profissionais foram mencionadas com maior frequência nos atendimentos: a psicologia e o serviço social. Destaca-se que, na saúde mental, o termo "papéis" refere-se às práticas profissionais específicas de cada categoria, regidas pelas leis do exercício profissional, dos conselhos de classe, entre outros, estando sujeitos a normas, direitos e deveres. Em contrapartida, o termo "funções" inclui as ações sociais e os diversos processos realizados pelos profissionais com o intuito de reinserir a pessoa com transtorno mental e o usuário de substância psicoativa na família e na sociedade ${ }^{14}$.

Sobre os papeis e as funções dos profissionais da rede psicossocial, um estudo realizado em sete serviços da RAPS, em Natal, apontou que as principais atividades realizadas pelos profissionais de nível superior foram: observação, registro de condutas e promoção da autonomia do usuário ${ }^{14}$. No entanto, o atendimento aos familiares e a capacitação especializada apresentavam-se inadequados entre os profissionais da equipe, o que pode gerar reprodução de condutas manicomiais e insegurança para que a equipe desenvolva ações dentro do modelo psicossocial ${ }^{14}$.

Em relação à falta de representatividade do enfermeiro nas falas dos entrevistados, supõe-se que, pela característica de sua formação acadêmica, é necessário investir mais nas ações desses profissionais nos cenários de saúde mental. Por isso, os cursos de Enfermagem precisam integrar-se aos serviços de saúde mental, de forma a atuarem coletivamente nas atividades do cotidiano, para que os graduandos possam vivenciar as práticas que se apresentam em campo ${ }^{15}$.

A visita domiciliar foi apontada por ambos os grupos de interesse como um meio de acolhimento e de assistência ao usuário e à família. Assim, é compreendida como importante ferramenta para o cuidado, proporcionando observação da rotina familiar e acompanhamento do usuário na área de abrangência, além de auxiliar na busca de faltosos nas atividades do serviço. O conhecimento do contexto e da rede de apoio local qualifica o atendimento prestado pelo serviço, possibilita a realização de abordagens condizentes com a realidade da família e busca compreender e auxiliar as demandas e as necessidades de saúde e de cuidado 9 .

No entanto, a visita domiciliar é uma alternativa que precisa ser melhor explorada pelos serviços, pois necessita de profissionais capacitados e de equipes preparadas para o cuidado contínuo do serviço aos domicílios. Para atingir esse atendimento integral como uma das dimensões da qualidade do cuidado, é necessário estabelecer uma rede de atenção que seja capaz de entender a complexidade do cuidado e de assegurar a promoção de autonomia e de cidadania às pessoas em sofrimento mental ${ }^{8}$.

No que se refere ao atendimento das famílias de usuários que frequentam o CAPS ad, percebe-se que o horário de funcionamento expandido do serviço avaliado contribui para o acesso a esses serviços. Estudo realizado em outro serviço da mesma modalidade, no qual o horário de funcionamento era de dez horas diárias, demonstrou maior presença de familiares e melhor atendimento das crises ${ }^{16}$. Outro estudo aponta que, para maior participação dos familiares, é preciso proporcionar disponibilidade de horários alternativos de atendimentos, como forma de facilitar o acesso, estimulando a inserção no serviço e a contribuição no processo terapêutico do usuário ${ }^{5,9}$.

Quanto ao apontamento sobre a localização do serviço, vale destacar que sua proximidade com locais de venda e de uso de drogas, característica vista pelos participantes como uma fragilidade, pode ser entendida como uma facilidade de acesso aos usuários que frequentam esses locais para decidirem pelo tratamento. E é exatamente nesse sentido que é objetivado, na portaria de implantação do CAPS AD IV, que ele deve estar localizado próximo aos locais onde ocorre o consumo de drogas, para acolher os indivíduos que procuram tratamento espontaneamente ou que são encaminhados ${ }^{1}$.

Entretanto, além da localização geográfica do serviço, outros fatores devem ser considerados para a adesão de usuários e de famílias ao tratamento, como a escolaridade, a eficácia e o apoio do CAPS, a divisão das atividades do cuidado no seio familiar, os agravos de saúde e os sentimentos de sobrecarga dos familiares. Dessa forma, entende-se que o CAPS exerce um papel importante na vida dessas pessoas, influenciando significativamente sua qualidade de vida ${ }^{17}$.

Ressalta-se, ainda, a necessidade do fortalecimento das ações de saúde mental no âmbito da atenção primária e articuladas em rede para fortalecer a adesão e o acesso dos usuários ao serviço. Os cuidados de saúde mental prestados nesse nível de atenção podem minimizar o estigma e a discriminação, assim como auxiliam a respeitar os direitos humanos preconizados pela reforma psiquiátrica. Nesse sentido, salienta-se que a atenção primária favorece o vínculo entre usuário e equipe de saúde, descentralizando o saber médico e estimulando a participação dos usuários, o que gera resultados positivos ${ }^{18}$.

Um estudo realizado com equipes de referência de matriciadores e de gestores, no Sudeste brasileiro, demonstra que o matriciamento em saúde mental torna-se uma importante ferramenta de transformação para os profissionais do CAPS, pois eles se aproximam das discussões a respeito da área de abrangência, bem como para os profissionais da atenção primária, que se familiarizam com a temática da saúde mental. Esse estudo destaca, ainda, a redução dos medos, principalmente de profissionais das UBS, em lidar com as pessoas em sofrimento psíquico, e a aproximação entre UBS e CAPS, em uma troca produtiva de saberes e práticas ${ }^{19}$.

Outra potencialidade citada pelos entrevistados diz respeito ao acolhimento e à humanização dos profissionais durante os atendimentos. Entende-se que a conduta da equipe está diretamente relacionada à eficácia do tratamento, visto que a relação de cada profissional com o usuário e seus familiares deve ser singular e deve permitir que as possibilidades de ajudar o sujeito se multipliquem. Para isso, as relações interpessoais da equipe junto aos usuários e aos familiares devem resultar no cuidado acolhedor, resolutivo e humano ${ }^{13}$. 
Além disso, um estudo que avaliou a adesão de usuários aos serviços de saúde mental menciona como potencialidade do serviço o vínculo estabelecido com os profissionais, o acolhimento, a escuta e a singularidade do projeto terapêutico ofertado. Compreende-se que o acolhimento constitui ferramenta primordial para aumentar o vínculo entre profissionais e usuários, possibilitando melhor compreensão da doença e resultando em autonomia para o cuidado 6 .

Os grupos de apoio, direcionados aos familiares, proporcionam aos participantes compartilhar as vivências da dependência química, fortalecendo e auxiliando na tomada de decisão. Tal fato pode ser corroborado com um estudo realizado no Rio Grande Sul, em que os familiares reconhecem mudanças de suas atitudes com relação à dependência química e ao entendimento sobre as questões comportamentais do usuário. Apontou-se como positivas as orientações recebidas pelos profissionais, no sentido de contribuir para o estabelecimento de um ambiente familiar integrado e menos fragmentado 5 .

Além do apoio familiar e da equipe multiprofissional já citado, evidencia-se o protagonismo dos usuários no reconhecimento das mudanças necessárias para a eficácia do seu tratamento. Com isso, o usuário identifica o uso compulsivo das drogas com os fatores desagradáveis vivenciados, para a conscientização dos prejuízos da vida, seja na própria qualidade de vida seja na de sua família 6 .

Ainda de acordo com o estudo, foi consenso entre os entrevistados que o usuário precisa responsabilizar-se pelo seu próprio tratamento. Nessa perspectiva, é importante salientar o vínculo entre as equipes de saúde mental e o usuário, de modo a proporcionar o diálogo e o acesso a todos os pontos de atenção da RAPS ${ }^{6}$. Embora os CAPS sejam serviços específicos para o cuidado em saúde mental, o ideal é que ofereçam o tratamento por um período transitório, e não por longos períodos, pois seu objetivo é fortalecer o cuidado em rede, estreitar vínculos com a atenção primária e com os demais pontos de atenção ${ }^{20}$.

Portanto, as ações e as práticas de cuidado em saúde mental devem ser vistas como uma oportunidade de alcance à autonomia do usuário, à inclusão social e ao suporte emocional nos processos da própria vida. Assim, tais práticas devem ter como foco o contexto no qual o usuário encontra-se inserido ${ }^{20}$. Nessa lógica, a reabilitação psicossocial deve preconizar espaços de trocas, valorizando a subjetividade e o protagonismo do usuário ${ }^{20}$.

\section{CONCLUSÃO E IMPLICAÇÕES PARA A PRÁTICA}

Esta pesquisa oportunizou avaliar as ações ofertadas pelo CAPS ad, a partir da avaliação de Quarta Geração, metodologia que permite considerar as diferentes perspectivas dos grupos de interesses, compostos neste estudo pelos familiares e pelos usuários do serviço.

Dessarte, evidenciou-se que as ações ofertadas pelo CAPS ad em estudo são reconhecidas positivamente pelos familiares e pelos usuários do serviço, mesmo diante das fragilidades identificadas pelos participantes, tais como a localização geográfica do CAPS ad e o fluxo fragmentado nos atendimentos da RAPS, demonstrando a necessidade de adequações na rede de cuidados psicossociais.

O serviço foi considerado de fundamental importância pelos grupos de interesse devido à integralidade e à gratuidade no tratamento e no fornecimento das medicações, assim como ao atendimento com a equipe multiprofissional e à disponibilidade de horário estendido sem prejuízos das atividades profissionais aos frequentadores. Também foi destacada a humanização e o acolhimento, o apoio emocional e as orientações e as trocas de informação fornecidas pelos profissionais para a compreensão da dependência química como doença e para a ênfase na corresponsabilização do usuário ao seu tratamento.

Com relação às implicações para a prática de Enfermagem, o estudo possibilitou reconhecer a eficácia do CAPS ad como um serviço especializado no cuidado à pessoa em uso de álcool e de outras drogas. No entanto, a partir da avaliação dos familiares e dos usuários, percebe-se a importância de aprimorar a articulação das ações interprofissionais e intersetoriais entre 0 CAPS ad e os demais pontos de atenção, assim como a inclusão da família como uma aliada para a continuidade do tratamento.

Evidencia-se que o CAPS ad consiste em um serviço especializado de saúde mental, de base territorial, pertencente a RAPS, com foco na reinserção social dos usuários na comunidade. Fazem-se necessários novos estudos que investiguem os diferentes cenários nos quais o cuidado em saúde mental esteja inserido, a fim de qualificar o atendimento aos familiares e aos usuários que fazem uso de álcool e de outras drogas. Ressalta-se como limitação deste estudo a ausência da negociação entre os grupos de interesse em virtude da pandemia de Covid-19, o que impossibilitou o consenso e a aquisição de novas informações, compreendida como etapa de validação dos dados pelos participantes da pesquisa.

\section{FINANCIAMENTO}

Este trabalho foi realizado com apoio da Coordenação de Aperfeiçoamento de Pessoal de Nível Superior - Brasil (CAPES) - Código de Financiamento 001, na modalidade de bolsa de Mestrado, concedida a Paula Antunes Bezerra Nacamura, no período de 2019 e 2020

\section{CONTRIBUIÇÕES DOS AUTORES}

Desenho do estudo. Valéria Cristina Christello Coimbra. Maria Aparecida Salci. Marcelle Paiano

Coleta ou produção dos dados. Valéria Cristina Christello Coimbra. Maria Aparecida Salci. Marcelle Paiano. André Estevam Jaques. Mauro Belino Piratelli Filho. Jéssica dos Santos Pini. Camila Harmuch.

Análise de dados. Valéria Cristina Christello Coimbra. Maria Aparecida Salci. Marcelle Paiano. André Estevam Jaques. Mauro Belino Piratelli Filho. Jéssica dos Santos Pini. Camila Harmuch. 
Interpretação dos resultados. Valéria Cristina Christello Coimbra. Maria Aparecida Salci. Marcelle Paiano. André Estevam Jaques. Mauro Belino Piratelli Filho. Jéssica dos Santos Pini. Camila Harmuch.

Redação e revisão crítica do manuscrito. Paula Antunes Bezerra Nacamura. Maria Aparecida Salci. Valéria Cristina Christello Coimbra. André Estevam Jaques. Mauro Belino Piratelli Filho. Jéssica dos Santos Pini. Camila Harmuch. Marcelle Paiano

Aprovação da versão final do artigo. Paula Antunes Bezerra Nacamura. Maria Aparecida Salci. Valéria Cristina Christello Coimbra. André Estevam Jaques. Mauro Belino Piratelli Filho. Jéssica dos Santos Pini. Camila Harmuch. Marcelle Paiano

Responsabilidade por todos os aspectos do conteúdo e a integridade do artigo publicado. Paula Antunes Bezerra Nacamura. Maria Aparecida Salci. Valéria Cristina Christello Coimbra. André Estevam Jaques. Mauro Belino Piratelli Filho. Jéssica dos Santos Pini. Camila Harmuch. Marcelle Paiano

\section{EDITOR ASSOCIADO}

Maria Catarina Salvador da Motta (๑)

\section{EDITOR CIENTÍFICO}

Ivone Evangelista Cabral $\odot$

\section{REFERÊNCIAS}

1. Portaria $n^{\circ} 3.588$, de 21 de dezembro de 2017 (BR). Altera as Portarias de Consolidação $n^{\circ} 3$ e $n^{\circ} 6$, de 28 de setembro de 2017, para dispor sobre a Rede de Atenção Psicossocial, e dá outras providências. Diário Oficial da União, Brasília (DF), 2017

2. Soares RH, Oliveira MAF, Pinho PH. Avaliação da atenção psicossocial em álcool e drogas na perspectiva dos familiares dos pacientes. Psicol Soc. 2019;31:e214877. http://dx.doi.org/10.1590/1807-0310/2019v31214877.

3. Ministério da Saúde (BR). Nota Técnica n. 11/2019: Esclarecimentos sobre as mudanças na Política Nacional de Saúde Mental e nas Diretrizes da Política Nacional sobre Drogas. Brasília: Ministério da Saúde; 2019

4. Guimarães TA, Rosa LCS. A remanicomialização do cuidado em saúde mental no Brasil no período de 2010-2019: análise de uma conjuntura antirreformista. O Social em Questão. 2019 [citado 2020 jan 10];22(44):111-38. Disponível em: http://osocialemquestao.ser. puc-rio.br/media/osq_44_art5.pdf

5. Nacamura PAB, Marcon SS, Paiano M, Salci MA, Radovanovic CAT, Rodrigues TFCS et al. Guidelines to the families of mental health service users from the multi-professional team's perspective. Rev Bras Enferm. 2020;73(Suppl 1):e20200389. http://dx.doi.org/10.1590/0034-71672020-0389. PMid:33295437.

6. Duarte MLC, Pereira LP, Carvalho J, Olschowsky A. Evaluation of families of crack users in relation to support groups. Rev Bras Enferm.
2018;71(Suppl5):2184-90. http://dx.doi.org/10.1590/0034-7167-20170808. PMid:30365782.

7. Paiano M, Kurata VM, Lopes, APAT, Batistela G, Marcon SS. Fatores intervenientes na adesão ao tratamento de usuários de drogas atendidos no CAPS-Ad. Rev Pesqui Cuid Fundam. 2019;11(3):687-93. http:// dx.doi.org/10.9789/2175-5361.2019.v11i3.687-693.

8. Terra MG, Mello AL, Siqueira DF, Beck CLC, Marinho LCP, Tisott ZL. Centro regional de referência de enfrentamento ao crack e outras drogas: relato de experiência. Ciênc Cuid Saúde. 2018;17(3):1-7. https://doi. org/10.4025/cienccuidsaude.v17i3.40881.

9. Moreira DJ, Bosi MLM. Qualidade do cuidado na Rede de Atenção Psicossocial: experiências de usuários no Nordeste do Brasil. Physis. 2019;29(02):e290205. http://dx.doi.org/10.1590/s0103-73312019290205.

10. Duarte MLC, Pereira LP, Olschowsky A, Carvalho J. Avaliação de quarta geração: atendimento aos familiares de usuários de crack. Rev Enferm UFSM. 2019;9(e12):1-20. http://dx.doi.org/10.5902/2179769229057.

11. Guba E, Lincoln Y. Avaliação de quarta geração. Campinas: Editora Unicamp; 2011.

12. Gonçalves LLM, Campos RTO. Narrativas de usuários de saúde mental em uma experiência de gestão autônoma de medicação. Cad Saude Publica. 2017;33(11):e00166216. http://dx.doi.org/10.1590/0102311x00166216. PMid:29166486.

13. Silva FS, Ferreira CB, Santos YLQ. Descrições do processo saúdedoença-cuidado na perspectiva de usuários de um centro de atenção psicossocial. REFACS. 2018;6(4):745-52. http://dx.doi.org/10.18554/ refacs.v6i4.3290.

14. Diniz AM. Projeto terapêutico singular na atenção à saúde mental tecnologias para o sujeito em crise. Sanare, Sobral [Internet]. 2017; [citado 2020 jan 10];16(1):7-14. Disponível em: https://sanare.emnuvens. com.br/sanare/article/view/1088/599

15. Santos RCA, Pessoa JM Jr, Miranda FAN. Rede de atenção psicossocial: adequação dos papéis e funções desempenhados pelos profissionais. Rev Gaúcha Enferm. 2018;39:e57448. http://dx.doi.org/10.1590/19831447.2018.57448. PMid:30043945.

16. Silva FP, Siqueira DF, Marchiori MRCT, Colomé JS. Abordagens teórico-práticas e metodológicas na formação em enfermagem para a saúde mental. Rev Enferm UFSM. 2020;10(e77):1-18. http://dx.doi. org/10.5902/2179769240141.

17. Pinho LB, Siniak, DS, Silva AB, Araújo LB, Folador B. Funcionamento de um centro de atenção psicossocial para o atendimento a usuários de crack. Rev Pesq: Cuid Fundam. Online. 2017;9(4): 1099-1106. http:// dx.doi.org/10.9789/2175-5361.2017.v9i4.1099-1106.

18. Kantorski LP, Jardim VMR, Treichel CAS, Mola CL, Alves PF, Ferreira $\mathrm{RZ}$ et al. Fatores associados a uma pior avaliação da qualidade de vida entre familiares cuidadores de usuários de Centros de Atenção Psicossocial. Cad Saude Colet. 2017;25(4):460-7. http://dx.doi. org/10.1590/1414-462x201700040327.

19. Bigatão MR, Pereira MB, Campos RTO. Ressignificando um Castelo: um Olhar sobre Ações de Saúde em Rede. Psicologia (Cons Fed Psicol). 2019;39:e185242. http://dx.doi.org/10.1590/1982-3703003185242.

20. Iglesias A, Avellar LZ. Matriciamento em Saúde Mental: práticas e concepções trazidas por equipes de referência, matriciadores e gestores. Ciênc saúde coletiva. 2019;24(4):1247-54. http://dx.doi. org/10.1590/1413-81232018244.05362017.

\footnotetext{
a Artigo extraído da Dissertação de Mestrado "Avaliação de Quarta Geração em um Centro de Atenção Psicossocial Álcool e Drogas", de autoria de Paula Antunes Bezerra Nacamura, orientada por Marcelle Paiano, Programa de Pós-Graduação em Enfermagem da Universidade Estadual de Maringá, defesa no ano de 2021.
} 